\title{
Author Index Volume 11, 1998
}

\section{Key to Abbreviations}

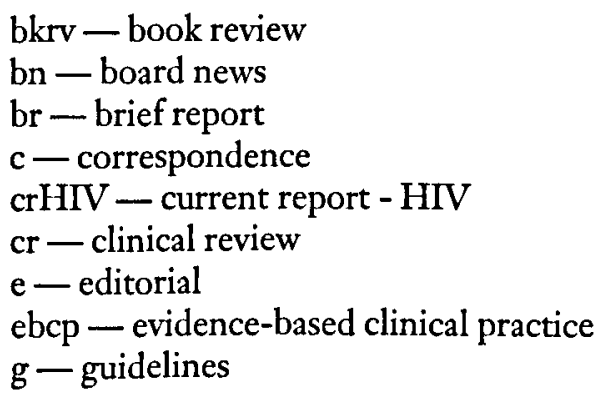

Abby, Stacey L. 391-step

Ackermann, Richard J. 366-cr

Acosta, David A. 425-bkrv

Anapol, Howard 434-oa

Andrews, Scott K. 291-mp

Avant, Robert 420-bn

Balano, Kirsten B. 158-crHIV

Baldwin, Laura-Mae 357-oa

Barber, L. Thomas 96-oa

Beasley, John 399-sp

Benjamin, Brian J. 12-oa, 250-c

Benjamin, Mignon 12-oa, 250-c

Berg, Alfred O. 216-ebcp

Bergman, James J. 498-bkrv

Berman, Brian M. 272-oa

Blackman, James R. 167-e

Blanc, Paul D. 66-br, 497-c

Block, Bruce 1-oa

Blount, B. Wayne 321-br, 459-mp

Bonfiglio, Mark F. 233-br

Branham, Ruth A. 1-oa

Brill, Patricia A. 445-oa

Brummel-Smith, Kenneth 242-e

Bunner, Scott H. 207-mp

Burke, George J. 170-c

Bystrisky, Alexander 282-cr

Carek, Peter J. 173-bkrv, 291-mp

Carmichael, Kevin 499-bkrv

Cate, Sara 84-bkrv

Cattoi, Robert J. 490-bn

Chayen, Benjamin 414-br

Christman, Carrol 399-sp

Coole, David 116-oa

Costa, Anthony J. 315-br

Cox, Daniel F. 264-oa

Cree, Jonathan 307-step

Crouch, Michael A. 247-e hcs - health care system

$\mathrm{hp}$ - health policy

mp - medical practice

oa - original article

refl - reflections in family practice

sp - special communication

step - STEPped care

$\mathrm{w}$ - world perspective

c-correction

Damos, James R. 399-sp

Dascalu, Shimon 414-br

Davis, Leland J. 498-bkrv

DeGaetano, Joseph S. 296-ebcp

deGruy, Frank 499-bkrv

Deyo, Richard A. 465-ebcp

Diaz, Victor 434-oa

Dolan, Peter 341-oa

Dong, Betty J. 158-crHIV

Driscoll, Charles E. 83-bkrv, 500-bkrv

Drivdahl, Christine E. 193-oa

Eaton, Charles B. 180-oa

Ebell, Mark H. 170-c

Eberly, Shirley W. 341-oa

Edwards, Allen R. 497-c

Eggertsen, Sam C. 500-bkrv

Eiff, $M$. Patrice 251-bkrv

Eimer, Micah J. 180-oa

Eliason, B. Clair 478-br

Epperly, Ted D. 296-ebcp

Everett, James 154-br

Farley, Tillman 83-bkrv

Felz, Michael W. 170-c

Fields Karl B. 334-bkrv

Fields, Scott A. 84-bkrv, 173-bkrv

Fink, Kenneth S. 318-br, 382-ebcp

Force, Rex W. 57-step

Fordyce, Meredith A. 357-oa

Fox, Chester 171-c

tFrame, J. Sutherland 87-oa

Frame, Paul S. 77-e, 87-oa, 341-oa

Frey Keith A. 333-bkrv

Froom, Jack 12-oa, 250-c

Froom, Paul 12-oa, 250-c

Galvin, Mary Sue 116-oa

Gerson, Lowell W. 315-br

Geyman, John P. 46-ebcp, 382-ebcp

tDeceased. 
Gjerde, Craig L. 399-sp

Glick, Michael 434-oa

Goldschmidt, Ronald H. 158-crHIV

Goldstein, Linda A. 481-br

Golusinsky, Lawrence L. 459-mp

Gomez, Sandra 425-bkrv

Gordon, Larry 434-oa

Gottlieb, Mark 336-oa

Grant, Mark D. 187-oa

Greenhouse, David L. 445-oa

Greenspan, Deborah 434-oa

Griswold, Kim S. 171-c

Guthmann, Richard A. 23-oa

Gutknecht, David R. 237-br

Hanna, George 264-oa

Hannapel, Andrew C. 296-ebcp

Harel, Linda 414-br

Harris, Ila M. 391-step

Harris, Kevin M. 391-step

Hart, L. Gary 357-oa

Hartnoll, Susan M. 272-oa

Helwig, Amy L. 336-oa

Herold, Arthur H. 251-bkrv

Herter, Christian D. 327-e

Hess, Andrea 127-cr

Hicks, Paul 422-c

Hines, Candace 171-c

Hodges, Barbara 154-br

Hulisz, Darrell T. 233-br

Hume, Anne L. 145-step, 422-c

Hunt, Vincent R. 85-bkrv

Hunter, Melissa H. 224-step

Hynes, Denise 96-oa

Jaen, Carlos R. 171-c

Jenkins-Woelk, Laura D. 357-oa

Jerant, Anthony F. 296-ebcp

Jonas, Wayne B. 244-e

Jones, Thomas V. 207-mp, 474-br

Kahler, Lucinda 171-c

Kahn Arlo 334-bkrv

Kakaiya, Ram 152-br

Kaplan, Boris 414-br

Katon, Wayne 282-cr, 347-oa

Kay, Lawrence E. 252-oa

Kerle, Karen 172-c

Koeniger, Mark A. 175-oa

Kohli, Rakhi 341-oa

Kovatchev, Boris P. 264-oa

Kroonen, Lisa M. 63-br

Lawless, Cara 307-step

Lee, Makau 410-br
Lee, R. David 140-mp, 331-c

Legg, Jill J. 158-crHIV

Lessenger James E 335-bkrv

Lloyd, Andree J. 296-ebcp

Loxterkamp, David 487-refl

Luisi, Andrea 145-step, 422-c

Lyna, Pauline R. 96-oa

Macera, Carol A. 445-oa

MacFarlane, Lori L. 224-step

Madlon-Kay, Diane J. 173-bkrv

Magill, Michael K. 165-refl, 406-br

Majeroni, Barbara A. 127-cr

Mandanas Linda 171-c

Manyam Bala V. 171-c

Martin, Kevin B. 28-oa

Maze, Gregory L. 410-br

McBride, Patrick E. 72-e, 423-c

McConaghy, John R. 324-refl

McQuade, William 180-oa

Meador, Clifton K. 166-e

Mehl-Madrona, Lewis E. 105-oa

Mettee, Thomas M. 28-oa

Mgebroff, A. Earl 417-refl

Miller, Daniel R. 296-ebcp

Miller, Karl 452-oa

Miser, William F. 193-oa

Monroe, Alicia 180-oa

Morikawa, Masahiro 485-w

Murray, Richard D. 233-br

Norris Thomas E. 333-bkrv

Oshinowo, Abayomi G. 459-mp

Padilla, Adriana 259-oa

Patel, Krishna 378-mp

Payne, Thomas 116-oa

Phillips, Theodore J. 84-bkrv

Plane, Mary Beth 399-sp

Post, Douglas 452-oa

Prince, T. Scott 481-br

Probst, Janice C. 445-oa

Prudhomme, Janice C. 66-br

Quillian, Warren C. 264-oa

Rabinowitz, Howard K. 229-hp, 434-oa

Raine, Tina R. 357-oa

Reamy, Brian V. 34-cr

Reilly, D 272-oa

Rimer, Barbara K. 96-oa

Roberts, E. Dwain 406-br

Rodney, Wm. MacMillan 80-c, 492-e

Rog, John 41-mp

Rosenblatt, Roger A. 357-oa

Roy-Byrne, Peter 282-cr 
Ruane, Thomas J. 81-c

Ruiz, David R. 425-bkrv

Saal, Andrew D. 240-refl

Sadovsky, Richard 434-oa

Scarpinato, Len 80-c

Schafer, Sean 259-oa

Scheerer, Amy 481-br

Schell, Bruce 445-oa

Scherger, Joseph E. 70-refl

Schneider, John R. 474-br

Schuman, Bernard M. 170-c

Schutz, Maggie 399-sp

Shusterman, Dennis J. 66-br

Siegel, Michael J. 474-br

Sifri, Randa 434-oa

Simmons, Melissa M. 224-step

Singh, Betsy B. 272-oa

Singh, B. Krishna 272-oa

Solomon, Gil L. 331-c

Stein, James H. 72-e; 423-c

Stein, Murray 282-cr

Struttman, Tim 481-br

Sullivan, Sean D. 382-ebcp
Swain, Geoffrey R. 336-oa

Taplin, Stephen 116-oa

Terrenoire, Jennifer 96-oa

Thorndike, Frances P. 264-oa

Tiemstra, Jeffrey D. 378-mp

Tisovec, Richard W. 152-br

Tuggy, Michael L. 426-oa

Turner, Barbara 434-oa

Unutzer, Jurgen 347-oa

Videlefsky, Andrea 321-br

Wagner, Ed 116-oa

Walker, Edward A. 347-oa

Ward, Em 41-mp

Watsky, Jay G. 175-oa

Watson, George 96-oa

Whealton, Edward G. 200-cr

White, William G. 172-c

Williams, Robert L. 28-oa

Woods-Powell, C. T. 96-oa

Yarnall, Kimberly S. H. 96-oa

Zervanos, Nikitas J. 174-bkrv

Zweifler, John 259-oa 